\title{
A NOTE ABOUT THE TEXT
}

All spellings are as they appear in the manuscripts quoted. Some quotations have been given punctuation to replace spaces in the manuscript text. The citation of sources uses a short title with a full title given in the bibliography. Notes give the pages of the article cited and are often followed by a ', $\mathrm{p}-$-' giving the particular pages of interest. Not all individual items in larger collections have been separately noticed with a title in the bibliography; neither have some single manuscripts which are given headings and titles in the notes. They are simply too numerous but all the manuscript numbers have been given in the text and bibliography. All the dates begin the year at 1 January, as the Scots had done since 1600, and otherwise conform to the calendars in use in Britain - the Julian to 1752 and the Gregorian thereafter. 